\title{
Una propuesta didáctica diseñada para favorecer el aprendizaje de la Inducción Electromagnética básica y el desarrollo de competencias digitales
}

\author{
Bettina Bravo \\ CONICET, Departamento de Ciencias Básicas, Facultad de Ingeniería (U.N.C.P.B.A.), Olavarria, Buenos \\ Aires,Argentina.bbravo@fio.unicen.edu.ar \\ ORCID: https:/ / orcid.org/0000-0002-3941-0547 \\ María José Bouciguez \\ ECienTec, Departamento de Ciencias Básicas, Facultad de Ingeniería (U.N.C.P.B.A.), Olavarria, Buenos \\ Aires,Argentina.mjbouci@gmail.com \\ ORCID: https:// orcid.org/0000-0002-4038-135X \\ Mariné Braunmüller \\ Departamento de Ciencias Básicas, Facultad de Ingeniería (U.N.C.P.B.A.), Olavarria, Buenos Aires, \\ Argentina.mbraunmu@fio.unicen.edu.ar \\ ORCID: https:/ / orcid.org/0000-0001-6749-3768
}

[Recibido: 14 Mayo 2018. Revisado: 9 Octubre 2018. Aceptado: 10 Noviembre 2018]

Resumen: En este trabajo se presenta y describe una propuesta de enseñanza diseñada para favorecer el aprendizaje del fenómeno de inducción electromagnética (IE) como así también el desarrollo de competencias digitales generales. La misma fue diseñada teniendo en cuenta diversos aspectos, tales como: los lineamientos curriculares oficiales, los resultados de investigaciones sobre concepciones y razonamientos de estudiantes y las contribuciones relacionadas con la forma en que se aprende y se enseña ciencias, así como la potencialidad de las TIC para favorecer el aprendizaje deseado. La propuesta fue implementada en un curso con estudiantes de 17-18 años de edad y los resultados obtenidos permiten percibir que la secuencia ha contribuido a lograr una mejor comprensión de las ideas claves asociadas a la IE, a la vez que implicó a los estudiantes en el uso de distintos recursos tecnológicos y con ello el desarrollo de habilidades inherentes a dicha utilización.

Palabras clave: Propuesta didáctica; Enseñanza; Física; TIC; Inducción electromagnética.

A didactic proposal designed to favor the learning of the Electromagnetic Induction and the development of digital competences.

\begin{abstract}
This paper presents and describes a teaching proposal designed to favor the learning of the phenomenon of electromagnetic induction (EI) as well as the development of general digital skills. It was designed taking in account various aspects, such as: the guidelines official curricular, the results of research on conceptions and reasoning of students and the contributions related to how science is learned and taught, as well as the potentiality of ICT to favor the learning desired. The proposal was implemented in a course with students 17-18 years old and the results obtained allow perceive that the sequence has contributed to achieve a better understanding of the key ideas associated with EI, at the same time implied to the students in the use of different technological resources and with it the development of inherent abilities to such use.
\end{abstract}

Keywords: Didactic proposal; Teaching; Physics; ICT; Electromagnetic induction.

Para citar este artículo: Bravo. B., Bouciguez. M. J., Braunmüller. M. (2019) Una propuesta didáctica diseñada para favorecer el aprendizaje de la Inducción Electromagnética básica y el desarrollo de competencias digitales. Revista Eureka sobre Enseñanza y Divulgación de las Ciencias 16(1), 1203. doi: 10.25267/Rev_Eureka_ensen_divulg_cienc.2019.v16.11.1203 


\section{Introducción}

Transitando el siglo XXI es indudable que las tecnologías de la información y la comunicación (TIC) han cambiado el estilo de vida, el trabajo, los pasatiempos, las relaciones sociales y hasta la manera de procesar la información por parte de los ciudadanos y ciudadanas.

Coincidiendo con Marchesi (2008), “este desarrollo acelerado de la sociedad de la información está suponiendo retos, impensables hace unos años, para la educación y el aprendizaje. Tal vez lo más relevante sea que nos encontramos con una nueva generación de aprendices que no han tenido que acceder a las nuevas tecnologías, sino que han nacido con ellas y que se enfrentan al conocimiento desde postulados diferentes a los del pasado" (p.7). Y, precisamente por la amplitud de las repercusiones de las TIC, pueden ser impulsoras potenciales de un cambio metodológico en las clases en general (Coll 2008) y en las de las ciencias en particular (Valverde-Crespo, de Pro-Bueno y González-Sánchez, 2018).

En consonancia con ello, en las más recientes reformas educativas implementadas en países como Argentina (CFE No 330/17) y España (BOE 2015) se está impulsando el desarrollo de competencias digitales como una finalidad de la educación obligatoria, prescribiéndose su abordaje de manera integrada y articulada con contenidos de las distintas disciplinas.

Resulta entonces inminente realizar cambios metodológicos en los distintos espacios curriculares que conforman la educación obligatoria a fin de favorecer no solo los respectivos aprendizaje disciplinares sino también el desarrollo de esas competencias digitales, entendidas como $^{1}$ un conjunto de conocimientos, procedimientos y actitudes necesarios para la utilización creativa, critica, segura y estratégica de las TIC, que conlleve a alcanzar los objetivos a los que se enfrenta/o se le plantea/n. Desde esta perspectiva, un sujeto digitalmente competente será aquel que sea capaz de buscar, obtener, procesar y comunicar información; transformarla en conocimiento y aplicarlo para tomar decisiones y resolver problemas en contextos variados (de índole escolar, laboral, de ocio, de relevancia social...) eligiendo y utilizando (con autonomía, eficacia, responsabilidad, criterio y fundamento) distintas herramientas tecnológicas.

Así, la inclusión de las TIC en las clases de Física se vuelve hoy inminente e indiscutiblemente necesaria. La inserción criteriosa y fundamentada de las mismas no solo favorecería el desarrollo de competencias digitales generales sino el propio aprendizaje de los conceptos, modelos, leyes, teorías, procedimientos que conforman esta disciplina. En tal sentido Pontes Pedrajas (2005) afirma que los recursos digitales pueden convertirse en potentes recursos didácticos en la enseñanza de la Física dada su potencialidad para simular fenómenos naturales difíciles de observar en la realidad, representar modelos de sistemas físicos inaccesibles, registrar procedimientos experimentales, facilitar la organización y tratamiento de datos, propiciar distintos medios para comunicar ideas y resultados, entre otras. En la tabla 1 se enumeran algunos recursos digitales que podrían incluirse en las clases de Física, resaltándose su aptitud para favorecer el aprendizaje de contenidos, procedimientos y actitudes relacionadas con la ciencia en general y con esta disciplina en particular.

Definición elaborada a partir de lo propuesto por lo documentos curriculares mencionados y la revisión realizada por Valverde - Crespo, de Pro Bueno y González Sánchez (2018) y la propuesta de Area Moreira (2008). 
Tabla 1. Adaptada y adoptada de la propuesta por Pontes Pedrajas (2005)

\begin{tabular}{|c|c|c|}
\hline $\begin{array}{l}\text { Función a desarrollar por las } \\
\text { TIC en clases de Física }\end{array}$ & $\begin{array}{l}\text { Aprendizaje del saber de las } \\
\text { ciencias que las TIC podrían } \\
\text { favorecer }\end{array}$ & $\begin{array}{l}\text { Recursos digitales posibles de } \\
\text { utilizar }\end{array}$ \\
\hline $\begin{array}{l}\text { Facilitar el acceso a la información } \\
\text { en distintos } \\
\text { comunicacionales y } \\
\text { representacionales. }\end{array}$ & $\begin{array}{l}\text { Construcción de conocimiento } \\
\text { teórico (conceptos, leyes, modelos, } \\
\text { teorías). } \\
\text { Desarrollo de pensamiento crítico y } \\
\text { reflexivo para seleccionar, analizar, } \\
\text { interpretar la diversidad de } \\
\text { información y fuentes aportadas. }\end{array}$ & $\begin{array}{l}\text { Navegadores de Internet. } \\
\text { Enciclopedia Multimedia. } \\
\text { Videos - tutoriales. } \\
\text { Simulaciones. } \\
\text { Animaciones. }\end{array}$ \\
\hline $\begin{array}{l}\text { Simular experimentos. } \\
\text { Facilitar el registro, organización y } \\
\text { procesamiento de datos, a partir de } \\
\text { la construcción de tablas y/o } \\
\text { gráficos. } \\
\text { Facilitar la construcción de modelos } \\
\text { y la interpretación de la relación } \\
\text { entre el comportamiento que se } \\
\text { visualiza y las expresiones } \\
\text { matemáticas que modelan ese } \\
\text { comportamiento. } \\
\text { Facilitar la comunicación a partir de } \\
\text { distintos formatos. }\end{array}$ & 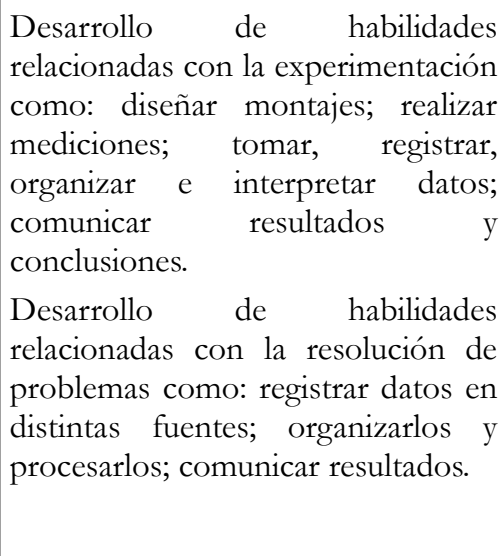 & $\begin{array}{l}\text { Procesadores de texto. } \\
\text { Hojas de cálculo. } \\
\text { Simulaciones } \\
\text { Laboratorios virtuales. } \\
\text { Graficadores. } \\
\text { Editores de animaciones y videos. } \\
\text { Herramientas de modelado } \\
\text { dinámico. } \\
\text { Editor de esquemas conceptuales. }\end{array}$ \\
\hline $\begin{array}{l}\text { Posibilitar el conocimiento de } \\
\text { múltiples y variadas situaciones, } \\
\text { problemas, contextos que denotan } \\
\text { la aplicación de las ciencias y la } \\
\text { tecnología. } \\
\text { Facilitar la comunicación y el } \\
\text { trabajo cooperativo. }\end{array}$ & $\begin{array}{l}\text { Desarrollo de actitudes positivas } \\
\text { hacia la ciencia, su producción y } \\
\text { aprendizaje. } \\
\text { Desarrollo de actitudes de respeto a } \\
\text { la diversidad de ideas y de una } \\
\text { conciencia intercultural. }\end{array}$ & $\begin{array}{l}\text { Navegadores de Internet. } \\
\text { Plataformas virtuales }-\quad \text { redes } \\
\text { sociales. }\end{array}$ \\
\hline
\end{tabular}

Incluir las TIC en el aula de Física entonces, propiciaría el alcance de un doble objetivo: favorecer el aprendizaje de esta disciplina y contribuir con el desarrollo de competencias digitales. Con estos objetivos en mente, se ha diseñado desde el programa IpACT: "Innovación Curricular para la Alfabetización Científico Tecnológica"2 diversas propuestas de enseñanza (véase por ejemplo Braunmüller, Bravo y Bouciguez 2017; Bravo, Bouciguez, Juárez, Montero e Inorreta 2018; Bravo, Juárez, Bouciguez, Montero e Inorreta 2016; Inorreta, Montero, Braunmüller, Bravo, Bouciguez y Sequeira 2017), una de las cuales se comparte en este trabajo.

Dicha propuesta está dirigida a alumnos y alumnas del último año de la Educación Secundaria (17 - 18 años de edad) con la cual se aborda el fenómeno de inducción electromagnética (IE). Coincidiendo con Almudí, Zuza y Guisasola (2016) el área de la inducción electromagnética es importante a nivel académico ya que se conjugan y sintetizan de forma creativa diferentes leyes de la electricidad y del magnetismo y la comprensión de este fenómeno permite a los ciudadanos tomar decisiones racionales sobre diversas aplicaciones tecnológicas presentes en su vida diaria. Esta importancia queda de manifiesta con el hecho que el fenómeno de

2 Programa de extensión universitaria ganador de una mención en la novena edición del Premio Clarín Zurich para la Educación. Conformado por un grupo de docentes-investigadores especialistas en la enseñanza de la Física, la Matemática y las TIC de la Facultad de Ingeniería de la Universidad Nacional del Centro de la Provincia de Buenos Aires (U.N.C.P.B.A.- Argentina) y docentes de escuelas secundarias. 
inducción electromagnética ocupa un lugar central en los diseños curriculares de educación media y universitaria (con orientaciones en ciencias naturales). A su vez, ha elegido esta temática porque los trabajos de investigación que se ocupan del aprendizaje logrado por los y las estudiantes en relación a la inducción electromagnética (IE) encuentran que los alumnos y las alumnas de distintos niveles educativos presentan serias dificultades para aprender las leyes que subyacen a ella (Braunmüller, Tenaglia y Bravo 2018; Almudí, Zuza, y Guisasola; 2016). En tal sentido, trabajos recientes como los realizados por Guisasola, Almudí, Zuza (2008); Guisasola, Almudí y Zuza (2010); Catalán, Caballero Sahelices, Moreira (2010); Almudí, Zuza, y Guisasola (2012); Almudí García, Ceberio Garate y Zubimendi Herranz (2013); Naizaque Aponte (2013); Tecpan, Benegas, Zabala (2015); muestran evidencias de las dificultades de aprendizaje y de la existencia de concepciones alternativas en las y los estudiantes en relación al fenómeno de IE, a saber:

- Presentan inconvenientes para imaginar y representarse mentalmente el fenómeno (la mayoría de las y los estudiantes están poco familiarizados con el fenómeno);

- Presentan dificultades para razonar en términos de las fuerzas que aparecen en la inducción electromagnética;

- Tienen problemas en la identificación de los factores asociados a la generación de una fem inducida: tendencia a asociar la IE con el campo magnético y/o flujo magnético (y no con la variación de flujo);

- No logran un aprendizaje comprensivo de la ley de Faraday: no le otorgan significado físico ni la aplican correctamente;

- Presentan dificultades para comprender entidades u objetos matemáticos (como integrales de superficie, volumen, trayectoria, los conceptos de flujo y circulación de un campo vectorial) que aparecen en la formalización de las leyes de IE y tienen además dificultad de aplicar en el contexto de las clases de Física, lo aprendido en las clases de Matemática.

La secuencia de enseñanza que se presenta en este trabajo tiene como objetivo ayudar a las y los alumnos a superar los obstáculos mencionados para lograr que:

- Reconozcan las variables involucradas y las condiciones que deben darse para que el fenómeno de inducción electromagnética se produzca y para que, a partir de él, se induzca una fem en un circuito eléctrico;

- Relacionen dichas variables a partir de la Ley de Faraday y la apliquen para resolver problemas significativos tecnológicamente.

A la vez y como ya se mencionó, con la propuesta diseñada se busca contribuir al desarrollo de habilidades y destrezas inherentes a la competencia digital, tales como identificar, almacenar, organizar y analizar información digital; comunicar en entornos virtuales de enseñanza y aprendizaje; crear $y$ editar contenidos nuevos en distintos formatos representacionales; integrar y reelaborar conocimientos y contenidos previos, usando recursos digitales; resolver problemas conceptuales a través de medios digitales (como las simulaciones y laboratorios virtuales) y usar herramientas de ofimática tales como procesadores de texto, hojas de cálculo, presentaciones, navegadores de internet, gestores de correo, considerados útiles para todo tipo de usuarios y usuarias de dispositivos digitales de procesamiento de datos (como la PC, tablet, celulares).

La propuesta de enseñanza que aquí se socializa como todas las propuestas diseñadas desde IpACT, se caracterizan desde tres aspectos teóricos centrales que se describen sintéticamente, a continuación 


\section{Marco teórico que subyace a la propuesta de enseñanza diseñada: breve síntesis}

- Concepción de aprendizaje: Se concibe al saber de la ciencia y al saber de las y los alumnos como dos modos de conocer. Dos maneras distintas de ver e interpretar el mundo que presentan características diferentes. Estas diferencias estarían relacionadas no sólo con el modelo explicativo; la idea, la concepción usada, sino también con su naturaleza representacional (Karmiloff-Smith 1992). En este sentido y atendiendo a lo que proponen distintos autores contemporáneos referentes de la psicología cognitiva (Chi, Roscoe, Slotta, Roy y Chase 2012; Pozo 2001; Vosniadou 2012); podemos decir que subyace al conocimiento intuitivo y a su construcción supuestos ontológicos, epistemológicos y conceptuales que suelen diferir de los que subyacen y caracterizan al saber científico. Desde esta perspectiva, el aprendizaje del saber de la ciencia no se basaría en la sustitución de una concepción por otra ni sólo en la multiplicación de las representaciones que el sujeto dispone para un dominio dado, sino en un cambio referente a esos principios que subyacen en la construcción del pensamiento cotidiano (Pozo 2001). Aprender ciencia implicaría no sólo comprender los conceptos, modelos, leyes y teorías propuestas sino también aprender a usar éstos de manera consciente, coherente y con consistencia al momento de resolver problemas, predecir e interpretar fenómenos; elaborar argumentaciones.

- La secuencia de enseñanza IDAS: La secuencia de enseñanza adoptada para favorecer un aprendizaje como el antes descripto, consta de cuatro fases: iniciación, desarrollo, aplicación y síntesis - conclusión (Bravo 2008).

La instancia de iniciación tiene como objetivo ayudar a las y los alumnos a reconocer qué piensan, cómo explican el fenómeno cuyo estudio se comienza a abordar, intentando así que lleguen a analizar y reflexionar acerca de cómo conocen y cuáles son las características primordiales de sus modos de conocer (relacionadas por ejemplo con sus contextos de usos, sus limitaciones explicativas). Esta instancia es fundamental porque será a partir de ese conocimiento que los y las alumnas construirán el conocimiento que se desea enseñar y porque deberán luego gestionar cognitivamente cuál de ellos usar para resolver las situaciones que se les presenten.

La instancia de desarrollo tiene como propósito realizar el abordaje formal del saber y saber hacer de la ciencia escolar, que conlleva distintos niveles de análisis y profundización. La instancia de aplicación tiene como objetivo favorecer el desarrollo de la habilidad de hacer un uso consistente y coherente del conocimiento construido. Se intenta así ayudar a las y los alumnos a que aprendan a elegir conscientemente de entre las múltiples representaciones que poseen, aquella que resulte más adecuada para resolver exitosamente un problema o para predecir o explicar una situación problemática.

La instancia de síntesis - conclusión intenta involucrar a los y las estudiantes en un proceso de concientización y explicitación de lo que aprendieron. Se espera que, guiados por el docente reflexionen sobre cómo aprendieron, distingan aquellas estrategias que les resultaron útiles aplicar para seguir aprendiendo y de lo que falta aún por aprender.

Esta secuencia didáctica sienta sus bases en los principios teóricos aportados por la piscología cognitiva contemporánea (antes descriptos) respecto de cómo se aprende ciencias y qué significa aprender y su impacto para favorecer el aprendizaje ha sido validado en distintos momentos y con diversas investigaciones realizadas por el grupo de trabajo (Bravo y Bouciguez 2018; Bravo y Pesa 2016; Juárez y Bravo 2015).

- La integración de las TIC como recurso didáctico central: Las propuestas diseñadas, integran las TIC con el fin de favorecer el aprendizaje de la Física y también el desarrollo de 
las competencias digitales antes mencionadas. Las herramientas informáticas se seleccionaron e incluyeron considerando como principales criterios las potenciales contribuciones que las mismas aportarían al logro de los objetivos planteados en relación al aprendizaje de los fenómenos a estudiar y la usabilidad, accesibilidad y diversidad de las mismas.

Los recursos que se incorporan bajo esta perspectiva son:

- Editor de mapas conceptuales, para la elaboración de esquemas conceptuales que realizan los alumnos y alumnas en las instancias didácticas de iniciación y de síntesis.

> Procesador de texto que se usa como soporte principal de la propuesta diseñada, la cual se comparte con los y las alumnas, mediante un entorno virtual de enseñanza y aprendizaje gratuito. Las consignas de la propuesta se presentan en formatos multimediales incluyendo hipervínculos y/o códigos QR para acceder a los distintos materiales digitales con los que se trabajan (vídeos, simulaciones y/o laboratorios virtuales, etc.). Los estudiantes deben utilizar el editor de texto para registrar sus respuestas de la misma manera, es decir, elaboradas en distintos formatos y compartidos a través de distintos canales virtuales de comunicación.

- Cámaras de fotos y videos para registrar no sólo imágenes sino también los procedimientos y datos surgidos de las experiencias realizadas.

$>$ Editores de fotos e imágenes, de videos y de imágenes animadas, buscando con ellos que las y los alumnos gestionen la articulación de diferentes lenguajes y soportes para dar cuenta de sus ideas, con creaciones propias que, como un todo, vinculen los registros y la explicación teórica que sustenta el trabajo que realizan.

- Planillas de cálculos para registrar, organizar en tablas y analizar los datos obtenidos en las experiencias.

- Simulaciones y laboratorios virtuales que permiten a los sujetos visualizar (en la pantalla de los dispositivos digitales) fenómenos y procesos físicos; interactuar, manipulando y transformando objetos en la interfaz de la aplicación y hasta realizar experiencias simuladas de difícil ejecución en el laboratorio. Existe un consenso creciente de que este tipo de recursos facilitarían el aprendizaje de la ciencia (Bouciguez y Santos 2008; Gómez Crespo 2008; Domínguez; 2010).

En el siguiente apartado describimos y ejemplificamos la secuencia de enseñanza diseñada.

\section{La propuesta de enseñanza}

La secuencia didáctica que aquí se presenta está conformada por 10 actividades mediante las cuales se aborda cuali y cuantitativamente, la ley de Faraday. Desde el punto de vista de la ciencia escolar se busca favorecer en los alumnos y las alumnas, la construcción de una idea que implica concebir que resulta posible inducir una fuerza electromotriz (fem) en un circuito cerrado, si se varía en el tiempo el flujo magnético que atraviesa dicho circuito. Variar el flujo magnético en el tiempo implica: variar la intensidad del campo magnético en el cual está inmerso el circuito, modificar el área que define el circuito, mover dicho circuito en una zona donde exista un campo magnético constante (o la combinación de todas estas acciones). La intensidad de la fem inducida resulta proporcional a la intensidad del cambio del flujo magnético en el tiempo (Ley de Faraday). Cabe aclarar que al referirnos a "circuito cerrado" también se contempla bajo esta expresión, la idea que el mismo sea únicamente una espira conductora, es decir que hace referencia a la necesidad de ofrecer una trayectoria cerrada para 
que se establezca la corriente generada a partir del establecimiento de la fem inducida por la variación del flujo magnético en el tiempo.

Por cuestiones de espacio, se presenta una actividad correspondiente a cada una de las fases antes comentadas, que guiaron el diseño.

\section{Actividades involucradas}

La primera actividad correspondiente a la instancia de iniciación, apunta a que los y las estudiantes reconozcan y expliciten sus ideas sobre el fenómeno que se comenzará a analizar. Las problemáticas planteadas (que están diseñadas para ser entregadas en forma separada y a medida que las y los alumnos vayan elaborando sus respuestas de manera individual) presentan distintos niveles de complejidad. El primer ítem demanda a los y las estudiantes explicitar sus ideas sobre el fenómeno de inducción electromagnética y los elementos necesarios para que se produzca. El segundo "exige" que piensen en la posibilidad de usar un imán (además de una espira cerrada) para lograr encender una lámpara y que expliciten como lo harían. En el tercero se les aporta evidencia sobre el fenómeno de inducción (simulado en el "Laboratorio electromagnético de Faraday" disponible en https://phet.colorado.edu/es/simulation/ legacy $/$ faraday $^{3}$ ) pidiéndoles que elaboren una explicación que involucra razonamientos cuali y cuantitativos.

Para favorecer la explicitación y comunicación de las ideas, se les solicita elaborar una respuesta en formato textual y confeccionar, luego un esquema conceptual haciendo uso del programa Cmap'Tools.

\section{Actividad 1: NUESTRAS IDEAS}

[1] En una clase de Física tu profesor construye un circuito como el de la figura y te pregunta si existe alguna manera de encender la lámpara sin utilizar una pila, batería o la red eléctrica. ¿Qué le responderías?

[2] Para ayudarte con tu problema te entrega alambre de cobre, una lámpara y un imán y te desafía a que con esos materiales logres encender la lámpara.

i. Propone cómo usarías los elementos disponibles para lograrlo.

ii. Explica la función que cumple cada elemento para hacer posible que la lámpara se encienda.

[3] Luego te muestra una experiencia simulada en un laboratorio virtual (disponible en https://phet.colorado.edu/es/simulation/faraday, solapa "Generador") y te solicita que:

i. Expliques por qué crees que en esas condiciones se enciende la lámpara.

ii. Decidas qué modificaciones le harías al circuito propuesto o al procedimiento empleado, a fin de lograr encender otra lámpara que requiere mayor diferencia de potencial para brillar. Justifica tu respuesta.

[4] A partir de las conclusiones arribadas elabora un esquema conceptual donde dejes de manifiesto los elementos necesarios para que una lámpara brille y la función que cumple cada uno, para que esto suceda. Para elaborar el esquema utiliza un Editor de esquemas conceptuales como el CmapTools disponible en https://cmaptools.softonic.com ó SimmpleMind Free Maps disponible en Play Store.

Durante la implementación de la propuesta el docente proyectó la simulación ante el gran grupo de alumnos. 
La actividad $\mathrm{N}^{\circ} 2$ (que se describe a continuación) pertenece a la instancia de desarrollo y tiene como objetivo que las y los alumnos reconozcan los elementos y condiciones que deben darse para que se produzca el fenómeno de IE.

Se elige trabajar en un laboratorio virtual dado que, si bien el fenómeno de IE puede observarse mediante experiencias sencillas, suele resultar difícil lograr (al menos en un laboratorio escolar) un montaje que permita modificar y medir la influencia de las variables de las que éste depende.

El recurso digital elegido (disponible en https://phet.colorado.edu/es/simulation/ legacy/faraday) permite justamente utilizar manipulables virtuales relacionados con las variables de las que depende el fenómeno (área de la espira; posición relativa espira - fuente de campo magnético; intensidad del campo magnético) y "visualizar" cómo ello afecta en la intensidad con que brilla la lámpara y/o valores indicados por el multímetro; lo que facilitaría la interpretación de las relaciones cuali y cuantitativas establecidas en la ley de Faraday.

\section{Actividad 2: ¡A EXPERIMENTAR!}

Entra en la simulación "Laboratorio electromagnético de Faraday" solapa bobina inducida (disponible en https://phet.colorado.edu/es/simulation/legacy/faraday). Interactúa con ella e intenta encender la lámpara. Manipula para ello todas las variables que la simulación te permite: posición del imán; posición del circuito; área de la espira; magnitud del campo.

[1] ¿Qué debe suceder con la intensidad del campo magnético, el área del circuito y la posición relativa imán - circuito para que la lámpara se encienda?

[2] ¿Qué debe suceder para que la luminosidad de la lámpara sea máxima?

[3] En base a lo observado concluye sobre:

i. Los elementos necesarios para encender una lámpara por inducción electromagnética.

ii. Las condiciones que deben darse para que la lámpara se encienda.

iii. Las condiciones que deben darse para que la lámpara brille con máxima luminosidad.

[4] Entra ahora a la solapa "generador" y en base a lo concluido, explica por qué se enciende la lámpara al hacer girar el imán (manteniendo constante: la posición relativa imán - circuito; el área del circuito y la intensidad del campo magnético).

[5] Faraday y Lenz fueron dos científicos que estudiaron el fenómeno de inducción electromagnética como el estudiado en la actividad anterior. Hallaron que: "la fuerza electromotriz inducida en un circuito resulta directamente proporcional al cambio en el tiempo del campo magnético que atraviesa el área delimitada por el circuito; o al cambio del área de dicho circuito y/o al cambio en la posición relativa fuente de campo - plano del circuito.” Escribe una expresión matemática que contemple estas observaciones.

La actividad denominada "Aplicando lo aprendido" pertenece a la instancia de aplicación. Esta actividad tiene como objetivo que las y los alumnos usen las ideas construidas para resolver distintas problemáticas que van desde los típicos ejercicios (que potencian el reconocimiento de variables y su vinculación en una expresión que describe matemáticamente el fenómeno físico) a problemas que gradualmente transitan desde los considerados cerrados hasta los más abiertos (este tipo de problemas se ejemplifican a continuación). 
Se propone en esta instancia el uso de medios audiovisuales para registrar y comunicar los resultados de las actividades realizadas. Se intenta así que los alumnos y las alumnas utilicen los formatos que habitualmente y de manera asidua manipulan en sus vidas cotidianas para registrar acontecimientos sociales (como son las fotografías y videos), en este caso para dar cuenta de los procedimientos empleados, los productos elaborados y la explicación teóricas subyacente.

\section{Actividad 5: APLICANDO LO APRENDIDO}

[1] Mira el video ${ }^{4}$ disponible en:

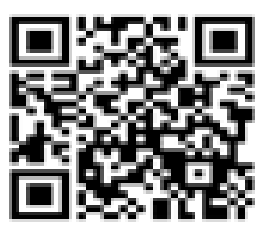

https://youtu.be/2hv2JN8d8OA

[2] Imita el procedimiento para lograr encender inalámbricamente un led.

[3] Registra tu procedimiento mediante un video y edítalo para usarlo como soporte que te permita explicar cómo es posible que el led se encienda.

[4] Diseña y construye un generador eléctrico.

[5] Registra fotográficamente el dispositivo e indica sobre la imagen los elementos utilizados.

[6] Elabora un informe donde expliques su funcionamiento y las variables que podrías manipular (y cómo deberías hacerlo) para lograr que la diferencia de potencial aportada por tu generador sea lo más grande posible.

La actividad denominada "Evaluando lo aprendido" pertenece a la instancia de síntesisconclusión. Esta actividad tiene como objetivo que los y las alumnas junto al docente evalúen qué y cuánto aprendieron y qué falta aún por aprender. Para ello se les propone leer las respuestas elaboradas en la primera actividad ("NUESTRAS IDEAS"), evaluarlas a la luz de sus nuevas ideas y de considerarlo necesario, ampliarlas y/o modificarlas. Así también y siempre con el fin de valorar lo aprendido, se les pide que resuelvan nuevas problemáticas relacionadas con la temática estudiada.

Los recursos digitales usados en esta instancia buscan incentivar y favorecer la explicitación de ideas. Para ello se plantean las problemáticas en distintos formatos (imágenes, videos, simulaciones) y se les solicita elaborar respuestas haciendo uso de distintos lenguajes (esquemas conceptuales, respuestas textuales; imágenes).

En esta instancia y al igual que en todas las anteriores, se propone el medio de dispositivos móviles para acceder a la información y/o comunicar respuestas a las problemáticas planteadas, intentando no solo continuar el desarrollo de competencias digitales (y con ello favorecer el uso creativo, critico, fundamentado de los recursos) sino también respetar en clases de Física el lenguaje y medios que los y las jóvenes usan actualmente para comunicarse entre ellos y acceder a la información.

\footnotetext{
4 Adoptado y adaptado de https://www.youtube.com/watch?v=rYzFEdgw6RY
} 


\section{Actividad N6: EVALUANDO NUESTRO APRENDIZAJE}

[1] Relee y evalúa las respuestas que elaboraste en la Actividad 1: NUESTRAS IDEAS y en base a todo lo aprendido evalúa la respuesta dada al punto 2 y el mapa entonces elaborado. De creerlo necesario modifica y/o amplía tus respuestas originales.

[2] El siguiente sitio puede visualizar un video que lleva el título de "Linterna Mágica". Accede a él y observa lo que ocurre.

[3] Emite una hipótesis sobre los materiales que conforman los elementos que constituyen el dispositivo e indícalos en el dibujo (que ha sido adoptado del disponible en el sitio mencionado).

[4] Explica cómo es posible que el led se encienda.

[5] Si deseas encender dos leds conectados en serie, ¿cómo modificarías tu dispositivo para que genere la energía necesaria? Justifica tu respuesta.

\section{Conclusiones}

Las innovaciones en las propuestas diseñadas reconocen como desafío de la educación, la formación integral de personas en habilidades y conocimientos que les permitan integrarse plenamente como sujetos pertenecientes y participantes activos de un contexto social y cultural en que las ciencias, las tecnologías y los medios de comunicación los atraviesan cada vez más en su hacer diario.

Con la propuesta de enseñanza diseñada y presentada en este trabajo se buscó favorecer no sólo la conceptualización del fenómeno de IE sino también el desarrollo de competencias digitales. Es por ello que se utilizó la secuencia IDAS como organizador de la propuesta de enseñanza y las TIC como principales mediadores buscando favorecer el alcance de los objetivos propios de cada instancia de la misma pero además implicando a los estudiantes en el uso de distintos recursos tecnológicos (para buscar, analizar, identificar, reflexionar, elaborar, comunicar, registrar, discutir, compartir ya sean datos, información, conocimientos y producciones).

Dicha propuesta ha sido implementada con dos grupos de alumnos del último año de educación secundaria ( $17-18$ años) en 8 encuentros de 2 horas cada uno. Tanto las y los alumnos como sus docentes, han evaluado satisfactoriamente la metodología implementada y su potencialidad para favorecer el aprendizaje de contenidos científicos y tecnológicos.

Del análisis de las respuestas que los y las estudiantes elaboraron antes y después de la implementación de la propuesta se pudo observar, en relación a la conceptualización del fenómeno de IE, que:

- En la instancia inicial, las y los alumnos no reconocen el fenómeno de IE. Así y ante la pregunta de si es posible encender una lámpara sin usar una pila, batería o la red domiciliaria, la gran mayoría de los alumnos responden que NO, porque falta la fuente de energía. Cuando se les propone pensar en la posibilidad de usar un imán, la mayoría plantea usarlo como la fuente necesaria (en la representación gráfica unen el cable a los 
extremos del imán y luego a la lámpara). Cuando se les presenta la evidencia empírica (simulada en el laboratorio virtual) la mayoría explica lo observado en términos de transformaciones energéticas (energía potencial - energía cinética - energía lumínica) pero sin explicitar cómo creen que la energía cinética se transforma en energía eléctrica. Ante la pregunta de qué modificarían en el circuito o procedimiento para logar que la lámpara brille en su máxima intensidad, las y los alumnos describen lo que la simulación les muestra (a mayor caudal de agua, mayor rapidez con la que gira la rueda y mayor luminosidad se observa en la lámpara) pero no explican lo observado.

- En la instancia final la mayoría de los alumnos y las alumnas reconocieron la posibilidad de generar una corriente eléctrica en un circuito cerrado, a partir del fenómeno de inducción electromagnética. Así también, un gran porcentaje de los y las estudiantes pudo aplicar la ley de Faraday para dar respuesta a problemas sencillos que implicaron resoluciones cuali y cuantitativas. Sin embargo, se observó que al momento de explicar las causas que conllevan al fenómeno de la IE, la mayoría centró su atención en la variación temporal del campo magnético por sobre la variación temporal del flujo magnético que atraviesa el circuito. Estas ideas construidas, correctas pero incompletas podrán servir de sólida base para construir otras más complejas y cercanas a las de la ciencia, conforme avancen en su nivel de escolarización. Esto nos advierte la necesidad de incluir en la propuesta diseñada, más actividades donde se presenten situaciones que permitan analizar y discutir la producción del fenómeno de IE como consecuencia de la variación del flujo magnético que atraviesa el circuito, haciendo hincapié en las distintas magnitudes de las que éste depende (como por ejemplo espiras girando en zonas donde existe un campo magnético estacionario o variando su área inmersas en dicho campo).

En relación al uso de los recursos digitales propuestos, se pudo observar que las y los alumnos utilizaron principalmente su teléfono móvil para acceder a la información propuesta en las actividades (mediante los códigos QR), elaborar los esquemas conceptuales y registrar los procedimientos experimentales (mediante fotografías y videos). En tanto las respuestas textuales las elaboraron en el formato tradicional de lápiz y papel (evidentemente les resulta más cómodo usar aún este recurso físico y no un procesador de texto, lo que podría deberse a que este lenguaje más tradicional es el que utilizan en el resto de las otras asignaturas). Solo cuando el recurso así lo demandaba (el uso del laboratorio virtual, por ejemplo) usan en lugar del teléfono, la PC.

Pero pese al uso cotidiano que los jóvenes dan a los dispositivos digitales se pudo observar que no les resultan conocidos los códigos QR y presentan dificultades al momento de responder y explicitar sus ideas y conocimientos mediante producciones digitales propias (editar y generar imágenes y videos, generar mapas conceptuales; respuestas textuales y/o incrustar imágenes en un procesador de texto, cargar datos en una planilla de cálculo y realizar gráficos a partir de los mismos).

Así las clases de Física se convirtieron en un espacio propicio no solo para aprender el saber y saber hacer que subyace a esta disciplina sino también uno a partir del cual, aprender a usar estos recursos digitales. Propuestas de este tipo entonces, favorecerían la tan ansiada alfabetización científica y digital, objetivos que hoy día persigue la enseñanza obligatoria.

\section{Agradecimientos}

A nuestras compañeras del proyecto IpACT de la Facultad de Ingeniería de Olavarría en el cual se enmarcó este trabajo y a directivos, docentes y alumnos y alumnas del Colegio Nuevas Lenguas y de la Escuela de Educación Secundaria $\mathrm{N}^{\circ} 6$. 


\section{Referencias}

Almudí García, J.; Ceberio Garate, M.; Zubimendi Herranz, J. (2013). Análisis de los argumentos elaborados por los estudiantes de cursos introductorios de física universitaria ante situaciones problemáticas pertenecientes al ámbito de la inducción electromagnética. Memorias del IX Congreso Internacional sobre Investigación en Didáctica de las Ciencias. Girona.

Almudí, J. M., Zuza, K., Guisasola, J., (2016). Aprendizaje de la teoría de inducción electromagnética en cursos universitarios de física general. Una enseñanza por resolución guiada de problemas. Enseñanza de las Ciencias, 34 (2)7-24.

Area Moreira, M. (2008). Innovación pedagógica con TIC y el desarrollo de las competencias informacionales y digitales. Investigación en la escuela, 64, 5-18.

Bouciguez, M.J., Santos, G. (2008). Simulaciones con computadora: abordaje didáctico y conceptos asociados. Memorias del I Congreso Internacional de Didácticas Especificas. Universidad Nacional de San Martín. Buenos Aires.

BOE (2015). Orden ECD/65/2015.

Braunmüller, M., Bravo, B. y Bouciguez, M. (2017). Las TIC como recurso central en la clase de física: la enseñanza de la energía mecánica. Publicado en las Memorias II Congreso Virtual Internacional de educación, innovación y TIC, INOVATIC 2017. Organizado por REDIME. Diciembre 2017. Madrid ISBN 978-94-92805-02-7

Braunmüller, M., Bravo, B. y Tenaglia, M. (2018). ¡Este tema es más difícil! Alumnas y alumnos con problemas en física. Trabajo presentado en las $3^{\circ}$ Jornadas de Enseñanza, Capacitación en Investigación en Ciencias Naturales y Matemática, a realizarse en la ciudad de Bernal, Buenos Aires. Setiembre de 2018.

Bravo B. (2008). La enseñanza y el aprendizaje de la visión y el color en educación secundaria. Universidad Autónoma de Madrid (Tesis doctoral sin publicar)

Bravo B., Bouciguez, M. (2018) Aprendiendo sobre la visión con las TIC como aliadas Alambique: Didáctica de las ciencias experimentales, 91, 12-19.

Bravo, B., Bouciguez, M., Juárez, A., Montero, M. y Inorreta, Y. (2016). La enseñanza de la física mediada por TIC. Publicado en actas 2do. Congreso Internacional de Enseñanza de las Ciencias y la Matemática 3er. Encuentro Nacional de Enseñanza de la Matemática. NIECyT. Fac. de Cs. Exactas. UNICEN

Bravo, B., Juárez, A., Bouciguez, M., Montero, M. y Inorreta Y. (2016) La enseñanza de la óptica mediada por tic. Una secuencia diseñada en la acción. En Pacheco, M. Bianchini, M. y Giamberardino, N. Articulando Universidad y Escuela Media. Editorial UNICEN. Tandil. Argentina. Libro impreso: ISBN 9789508584138

Bravo B., Pesa, M. (2016). El cambio conceptual en el aprendizaje de las ciencias. Un estudio de los procesos involucrados al aprender sobre la luz y la visión. Revista Electrónica de Enseñanza de las Ciencias). 15 (2) 258-280

Catalán, L.; Caballero Sahelices, C.; Moreira, M. A. (2010). Niveles de coceptualización en el campo conceptual de la Inducción electromagnética. Un estudio de caso. Lat. Am. J. Phys. Educ. 4 (1) 126-142.

Chi, M., Roscoe, R., Slotta, J., Roy, M., Chase, C. (2012) Misconceived Casual Explanations for Emergent Processes. Cognitive Science $36 \quad$ (1) 1-61 https://onlinelibrary.wiley.com/doi/pdf/10.1111/j.1551-6709.2011.01207.x 
Coll, C. (2008). Aprender y enseñar con las TIC: expectativas, realidad y potencialidades. En Cameiro, R., Toscano, J.C., Díaz, T. (coord.). Los desafíos de las TIC para el cambio educativo. Madrid: Metas Educativas 2021 de la OEI. Fundación Santillana.

Consejo Federal de Educación (2017). Resolución CFE ³30/17.

De Pro Bueno, A.J., Cañizares Millán, M. (2006). El uso de simulaciones en la enseñanza de la física. Alambique Didáctica de las Ciencias Experimentales. 50, 66-75.

Domínguez, M.A. (2010). Algunas consideraciones teóricas para caracterizar el proceso de enseñanza y aprendizaje en Tecnología Educativa y Conceptualización en Física. Estudios sobre interacciones digitales, sociales y cognitivas. Buenos Aires: UNCPBA.

Guisasola, J.; Almudí, J. M.; Zuza, K. (2008). Explicaciones de los estudiantes de primer curso de ingeniería sobre los fenómenos de inducción electromagnética. Revista de Enseñanz̧a de la Física. 21 (2) 33-47.

Guisasola, J.; Almudí, J. M.; Zuza, K. (2010). Dificultades de los estudiantes universitarios en el aprendizaje de la inducción electromagnética. Revista Brasileira de Ensino de Fisica, 32 (1) 1401-1/1041-9.

Inorreta, Y., Montero, M., Braunmüller, M., Bravo, B., Bouciguez, M. y Sequeira, A. (2017). Los circuitos eléctricos. una propuesta de enseñanza mediada por TIC. Congreso en Enseñanza de las Ciencias y la Matemática. Tandil, Agosto 2017

Jonassen, D, Carr, C. (2000). Mindtools: Affording Multiple Knowledge Representations for Learning en Computer as cognitive tools. Mahwah, NJ: Lawrence Erlbaum Assosiates.

Juárez, M., Bravo, B. (2015). Análisis de estrategias didácticas implementadas a fin de favorecer el aprendizaje de fenómenos ópticos. Revista Iberoamericana de Educación Didáctica de la Ciencias y de la Matemática. 69 (1).

Karmiloff-Smith (1992). Más allá de la modularidad. Madrid: Alianza Editorial.

Marchesi, A (2008). Preámbulo en Los desafíos de las TIC para el cambio educativo. Madrid: Metas Educativas 2021 de la OEI. Fundación Santillana.

Naizaque Aponte, N. (2013). Diseño de una estrategia didáctica para la enseñanza de la inducción electromagnética. Tesis de Maestría en Enseñanza de las Ciencias Exactas y Naturales Universidad Nacional de Colombia. Facultad de Ciencias. Bogotá, Colombia. http://bdigital.unal.edu.co/39628/1/1186696.2013.pdf

Pontes Pedrajas, A. (2005). Aplicaciones de las tecnologías de la información y de la comunicación en la educación científica. Primera parte: funciones y recursos. Revista Eureka sobre Enseñanza y Divulgación de las Ciencias, 2 (1) 2- 18.

Pozo, J.I. (2001). Humana mente. El mundo, la conciencia y la carne. Madrid: Ed. Morata.

Tecpan, S.; Benegas, J., Zabala, G. (2015). Entendimiento conceptual y dificultades del aprendizaje de electricidad y magnetismo identificadas por profesores. Lat. Am. J. Phys. Educ. 9 S1204-1/S1204-11.

Valverde-Crespo, D.; de Pro-Bueno, A., González-Sánchez, J. (2018). La competencia informacional-digital en la enseñanza y aprendizaje de las ciencias en la educación secundaria obligatoria actual: una revisión teórica. Revista Eureka sobre Enseñanza y Divulgación de las Ciencias, 15 (2) 2105-1/2105-15. 
Vosniadou, S. (2012) Reframing the Classical Approach to Conceptual Change: Preconceptions, Misconceptions and Synthetic Models en Second International Handbook of Science Education (1), 119-130. London: Springer

Zuza, K.; Almudí, J. M.; Guisasola, J. (2012). Revisión de la investigación acerca de las ideas de los estudiantes sobre la interpretación de los fenómenos de Inducción electromagnética. Revista Enseñanza de las Ciencias. 30 (2) 175-196.

Zuza, K.; Guisasola, J.; De Cock, M.; Bollen, L.; van Kampen, P.; Garzón, I. (2017). ¿Qué entienden los estudiantes universitarios de física general por fuerza electromotriz? Un estudio en cuatro países. X Congreso Internacional sobre Investigación en Didáctica de las Ciencias. Sevilla. 